\title{
Análise quantitativa da intensidade de riscos em projetos de smart grid no setor privado brasileiro
}

Quantitative analysis of risk intensity in smart grid projects in the brazilian private sector

Análisis cuantitativo de la intensidad del riesgo en proyectos de redes inteligentes en el sector privado brasileño

Recebido: 19/12/2021 | Revisado: 27/12/2021 | Aceito: 29/12/2021 | Publicado: 29/12/2021

\author{
Kleber Rocha de Oliveira \\ ORCID: https://orcid.org/0000-0002-1260-6363 \\ Universidade Estadual Paulista, Brasil \\ E-mail: kleber.oliveira@unesp.br \\ José Francisco Resende da Silva \\ ORCID: https://orcid.org/0000-0002-4671-0740 \\ Universidade Estadual Paulista, Brasil \\ E-mail: jose.resende@unesp.br \\ Fábio Luciano Violin \\ ORCID: https://orcid.org/0000-0001-9208-3082 \\ Universidade Estadual Paulista, Brasil \\ E-mail: fabio.violin@unesp.br
}

\begin{abstract}
Resumo
Esta pesquisa apresenta um estudo conceitual sobre as redes elétricas inteligentes (Smart Grid) e o gerenciamento de riscos de acordo baseado no guia Project Management Body of Knowledge (PMBOK) v. 6.0, assim como a elaboração de questionamentos acerca do gerenciamento de riscos. a metodologia aplicada foi uma survey utilizada para coleta de dados de profissionais de organizações do setor de energia elétrica que trabalham em projetos de redes elétricas inteligentes entregues ao setor privado. com a análise das respostas, concluiu-se que o planejamento do projeto pode ser considerado como uma fase crítica em relação ao gerenciamento de riscos do projeto, uma vez que pode apresentar maiores complicações caso não seja desenvolvida de forma satisfatória, prejudicando ainda o restante dos grupos de processos como a execução, o controle e monitoramento e o encerramento do projeto. além disso, as organizações em sua maioria realizam gerenciamento de riscos, mas nem sempre de forma frequente, completa e efetiva ou ainda utilizando técnicas e ferramentas altamente confiáveis e difundidas.
\end{abstract}

Palavras-chave: Gerenciamento de projetos; Gerenciamento de riscos; Redes elétricas inteligentes.

\begin{abstract}
This research presents a conceptual study on smart grid and risk management according to the Project Management Body of Knowledge Guide (PMBOK) v. 6.0, as well as the preparation of risk management agreement. the methodology applied was a survey used to collect data from professionals of electricity sector organizations that work on intelligent electric network projects delivered to the private sector. with the analysis of the responses, it was concluded that the project planning can be considered as a critical phase in relation to the project risk management, as it may present further complications if it is not developed in a satisfactory way, harming groups of processes such as implementation, control and monitoring and project closing. in addition, most organizations do risk management, but not always frequently, completely and effectively or still using highly reliable and diffused techniques and tools.
\end{abstract}

Keywords: Project management; Risk management; Smart grid.

\section{Resumen}

Esta investigación presenta un estudio conceptual sobre las redes inteligentes (Smart Grid) y la gestión de riesgos en consecuencia, basado en la guía del Cuerpo de Conocimiento de la Gestión de Proyectos (PMBOK) v. 6.0, así como la elaboración de preguntas sobre gestión de riesgos. La metodología aplicada fue una encuesta utilizada para recolectar datos de profesionales de organizaciones del sector eléctrico que trabajan en proyectos de redes eléctricas inteligentes entregados al sector privado. Con el análisis de las respuestas, se concluyó que la planificación del proyecto puede ser considerada como una fase crítica en relación a la gestión de riesgos del proyecto, ya que puede presentar mayores complicaciones si no se desarrolla satisfactoriamente, perjudicando al resto de los grupos de procesos. tales como ejecución, control y seguimiento de proyectos y cierre de proyectos. Además, la mayoría de las organizaciones realizan la gestión de riesgos, pero no siempre con frecuencia, de manera completa y eficaz o utilizando técnicas y herramientas altamente confiables y generalizadas.

Palabras clave: Gestión de proyectos; Gestión de riesgos; Redes eléctricas inteligentes. 


\section{Introdução}

Sendo um país com dimensões continentais e alto índice demográfico, o Brasil possui necessidades muito complexas para manter em equilíbrio a geração, transmissão e distribuição de energia elétrica (Gallotti, 2021). Somado a essa complexidade característica, há um crescente acesso a tecnologias e ao consumo de produtos, bens e serviços no país, resultando, portanto, no aumento da demanda energética e nos proporcionando maiores riscos de apagões (falta de energia), além dos tão frequentes roubos de energia, conhecidos popularmente como "gatos" (Guerra et al., 2010).

O conceito de Rede Elétrica Inteligente, conhecida como Smart Grid (SG), aborda diversas características inovadoras que visam aperfeiçoar o setor de energia elétrica através da aproximação entre as concessionárias e consumidores, da maior resistência a ataques físicos e cibernéticos, da redução dos impactos ambientais e a da capacidade de recuperar-se (Arruda e Oliveira, 2013), além da utilização de inteligência computacional (Costa et al, 2021).

Pereira e Weiss (2017), afirmam que quando são incorporadas as tecnologias à infraestrutura de sistemas de energia elétrica, há benefício não só para os consumidores, mas também para as distribuidoras. Essa incorporação permite que sejam alcançadas reduções de custos operacionais (leitura, cortes e religações realizadas remotamente), de gastos relativos ao pagamento de multas e reembolsos por conta das interrupções e danificações de equipamentos, bem como redução das perdas não técnicas. Em (Firmes \& Celeste, 2018), também se sugere uma infraestrutura mais tecnológica proporciona também maior precisão para identificação de falhas, interrupções, quedas de fio e o furto, entre outros.

Em 2015 as perdas financeiras com furto de energia ultrapassaram R\$ 8 bilhões, considerando as 59 principais distribuidoras de energia elétrica no Brasil (Secretaria de Infraestrutura e Meio Ambiente). Esse problema previsto e indesejado é um dos tópicos importantes da implantação do conceito de SG e através de medidores inteligentes, por exemplo, detectando e impedindo as fraudes, as concessionárias e consumidores adquirem maior autonomia, usando a eletricidade de forma racional, reduzindo o consumo de energia elétrica e, consequentemente, podendo reduzir as contas dos consumidores (Guerra et al., 2010).

Quando a rede elétrica convencional é comparada com a SG, percebe-se, por exemplo, a grande evolução referente às soluções de muitos problemas de gestão do sistema elétrico, uma vez que são tomadas decisões mais precisas a respeito dos recursos disponíveis e das manobras técnicas utilizadas (Oliveira et al., 2015).

Além de todas essas modificações, no futuro, com a implantação das SG, a compra de energia poderá ser feita de qualquer concessionária de distribuição interligada ao sistema e os custos reais de venda da energia gerada/transmitida serão monitorados e informados em tempo real aos consumidores, fazendo surgir assim novos modelos de mercado (Oliveira, Cleto e Seleme, 2015).

Destaca-se também a definição de projeto como um esforço temporário empreendido para criar um produto, serviço ou resultado único (PMI, 2017). Baseando-se nessa descrição, pode-se classificar a implantação de uma rede elétrica inteligente como um projeto que possui o objetivo de tornar o sistema elétrico mais autônomo e eficiente, na qual seu encerramento ocorre quando seus serviços e equipamentos estiverem implantados corretamente e funcionalmente. Então a gestão dos eventos envolvidos nas estratégicas na gestão de projetos, onde as incertezas são inevitáveis, podem auxiliar no gerenciamento proativo de projetos (Filippetto et al, 2021).

Como todo projeto, a implantação de uma SG também possui diversos riscos e desafios. No Brasil, o grau de complexidade é um pouco maior se comparado com países como Alemanha, EUA e Japão que já possuem um elevado investimento em tecnologia de comunicação, objetos inteligentes, possuem territórios menores que o Brasil, além de uma economia mais estável.

Dessa forma, quando se trata de uma SG, deve-se levar em consideração desde os riscos e desafios mais comuns encontrados em outros países até os riscos específicos baseados em nossa realidade nacional e regional. Este presente trabalho 
é resultado de um estudo sobre o gerenciamento de riscos em projetos de implantação de redes elétricas inteligentes do setor privado, baseando-se no guia Project Management Body of Knowledge (PMBOK®) - Guia do Conhecimento em Gerenciamento de Projetos da Project Management Institute - PMI, versão 6.0, para compreender como é realizado o gerenciamento de riscos nestas organizações, servindo como base para análise de propostas para que as redes elétricas inteligentes sejam melhores implantadas.

\section{Metodologia}

Para coleta dos dados foi utilizado o método de pesquisa Survey, na qual pode ser descrita como um método de investigação cuja finalidade é colher os dados de uma amostra representativa de uma população específica. Evitando então realizar o censo, ou seja, ouvir todos os indivíduos, este tipo de pesquisa sugere usar os resultados obtidos de forma generalizada ao universo da população escolhida (Babbie, 2005). Através então da coleta sistemática de informações, o intuito da pesquisa do tipo Survey é compreender e/ou prever alguns aspectos do comportamento da população em estudo.

Portanto, optou-se, pela aplicação de um questionário virtual para realizar a coleta dos dados, sendo que, de acordo com Silva et al (1998), o questionário é uma forma previamente estruturada e organizada de fazer a coleta de dados em uma população específica, buscando informações adicionais e complementares sobre um determinado assunto.

Dessa forma, foi construído um questionário online, estruturado e não disfarçado, onde os respondentes sabiam qual era o objetivo da pesquisa, e padronizado, usando principalmente questões fechadas. Além disso, durante a redação das questões, foi tomado o cuidado de utilizar uma linguagem clara, de fácil entendimento, com termos técnicos de conhecimento geral sobre gerenciamento de riscos para as organizações. Para evitar dúvidas, foi colocado as definições junto aos termos específicos de gerenciamento de riscos, de acordo com o Guia PMBOK (PMI, 2017).

As perguntas foram agrupadas e ordenadas em quatro blocos: frequência e forma de realização dos processos de gerenciamento de riscos nos projetos, intensidade dos riscos em cada um dos grupos de processos dos projetos, dados organizacionais e, por último, dados pessoais. Foram elaboradas instruções de preenchimento no início de cada seção.

O primeiro bloco é composto por sete perguntas fechadas tricotômicas e obrigatórias, uma para cada processo de gerenciamento de riscos, de acordo com o Guia do Conhecimento em Gerenciamento de Projetos: Planejar o Gerenciamento dos Riscos, Identificar os Riscos, Realizar a Análise Qualitativa dos Riscos, Realizar a Análise Quantitativa dos Riscos, Planejar as Respostas aos Riscos, Implementar Respostas a Riscos e Monitorar os Riscos.

Já a segunda seção contém uma única pergunta fechada obrigatória do tipo matriz, solicitando que seja classificada a intensidade dos riscos em cada um dos cinco grupos de processos de um projeto: Iniciação, Planejamento, Execução, Monitoramento e Controle, além do Encerramento.

Sobre os dados organizacionais, foram utilizadas duas perguntas abertas para identificar o nome da organização (pergunta opcional) e a função do respondente nessa organização (pergunta obrigatória), além de duas perguntas fechadas obrigatórias e de resposta única sobre o tipo de organização e domínio de negócio na qual a empresa atua. Ainda se utilizou de uma pergunta fechada obrigatória e de múltipla escolha sobre as áreas de atuação da organização, podendo ser selecionada uma ou mais opções neste último questionamento.

Por último, através dos dados pessoais dos respondentes, foi possível compreender e distinguir quem são os respondentes do questionário. Este bloco é formado por apenas três questões, uma aberta e opcional para o nome completo do respondente, contendo a instrução de que o nome será preservado e não constará no trabalho, uma pergunta fechada obrigatória e de resposta única sobre o estado da lotação e, por fim, uma pergunta fechada obrigatória e de múltipla escolha sobre o nível de formação acadêmica.

Após aplicação do questionário e antes da realização da análise dos dados, foi realizada a verificação e tabulação dos 
dados coletados. A verificação dos dados serve para analisar se todas as questões foram respondidas, se as respostas abertas estão escritas legivelmente e de forma compreensível, bem como se há coerência nas respostas. Já a tabulação significa organizar os dados em tabelas, para serem analisados por processo de técnica de análise estatística, podendo ser feita de forma manual, mecânica, eletrônica ou parcialmente manual e eletrônica. (Mattar, 1996; Marconi e Lakatos, 1996)

A tabulação dos dados obtidos nas perguntas fechadas foi realizada de maneira eletrônica utilizando a planilha eletrônica, um software gratuito e online oferecido pela Google em seu serviço GSuítes. Após tabulação foram elaborados diversos gráficos dos tipos pizza, colunas, barras e linhas para facilitar a análise e visualização dos resultados.

Análise de dados foi realizada baseada nas respostas ao questionário dos participantes e comparada com boas práticas encontradas nas referências utilizadas nesta pesquisa, principalmente as sugeridas no Guia PMBOK (PMI,2017). Todas essas análises são apresentadas em forma gráfica, seguidas de uma discussão crítica sobre a percepção dos resultados, apontando suas fragilidades e pontos importantes. Para facilitar a compreensão análise foi dividida em quatro partes de acordo com a sequência lógica das questões no questionário.

$\mathrm{Na}$ primeira parte é apresentado perfil dos participantes e informações de suas organizações a fim de dar uma personificação sobre suas qualificações, formação escolar, sua posição na organização onde trabalha, a região de sua lotação, além das áreas especificadas de atuação destas empresas e abrangência relativas ao mercado, sendo nacional e/ou internacional.

Na segunda parte foi feito uma análise dos resultados de forma específica abordando os processos que envolvem todo o gerenciamento de risco. Esses processos

envolvem o planejamento de gerenciamento de risco, o processo de identificação dos riscos, a análise qualitativa e quantitativa dos riscos, planejamento das respostas aos riscos, implementação de respostas aos riscos e o monitoramento dos riscos.

Na terceira parte da pesquisa foi feita uma análise dos resultados sobre cada um dos grupos de processos do gerenciamento de projeto, mostrando qual é a realidade gerencial e operacional, em relação a cada um dos grupos do ciclo de vida dos projetos. Pontualmente é analisada qual a percepção dos impactos, relativos a riscos, na fase da iniciação do projeto, do planejamento do projeto, da execução do projeto, do controle e monitoramento do projeto, e por fim, do encerramento do projeto.

Na quarta e última parte da pesquisa, foi realizada uma análise geral da intensidade dos riscos, abordando e discutindo como cada categoria de intensidade risco se manifesta longo das fases da gestão do projeto de acordo com a percepção da amostra participante. As categorias foram classificadas como: mínima ou inexistente, razoável ou média e intensa ou aguda.

\section{Resultados e Discussão}

O questionário finalizado foi enviado via internet para cerca de 125 (cento e vinte e cinco) profissionais especialistas do setor elétrico de organizações privadas brasileiras, obtidos por meio de pesquisas comuns na WEB e nas redes de contatos dos pesquisadores, além de ter sido divulgado via redes sociais com foco profissional. O intuito era compreender como e com que frequência o gerenciamento de riscos em projetos de redes elétricas no setor privado é realizado, bem como qual a percepção de intensidade de riscos em cada grupo de processo dos projetos. Ao final de 65 (sessenta e cinco) dias corridos, foram obtidas 110 (cento e dez) respostas, ou seja, aproximadamente $88 \%$ da amostra inicialmente selecionada.

Para apresentação da análise, a discussão foi estruturada em quatro segmentos: Análise do Perfil dos Respondentes e Organizações, Análise dos Resultados Específicos, Análise dos Resultados sobre os Grupos de Processos e Análise Geral da Intensidade dos Riscos ao longo do Projeto, na qual estão expostos a seguir os dados coletados e as discussões de acordo com cada segmento e resultados obtidos. Os resultados mais completos, por segmentação foram apresentados no SIMPEP 2021 
(Silva, Oliveira e Silva, 2021).

Baseando-se na forma de implementação e gerência dos projetos envolvendo energia elétrica dos entrevistados, foi solicitado que optasse pela opção correta dentre as três alternativas dispostas para cada um dos sete processos de gerenciamento dos riscos do projeto: Planejar do Gerenciamento dos Riscos, Identificar dos Riscos, Realizar Análise Qualitativa dos Riscos, Realizar Análise Quantitativa dos Riscos, Planejar das Respostas aos Riscos, Implementar das Respostas aos Riscos e Monitorar dos Riscos.

\subsection{Planejar do Gerenciamento dos Riscos}

O primeiro processo, de acordo com PMI (2017), Planejar do Gerenciamento dos Riscos é descrito como processo de definição de como conduzir as atividades de gerenciamento dos riscos de um projeto. Ao serem questionados sobre qual a frequência se da realização de um plano de gerenciamento de riscos, 63,6\% assinalaram que sempre há plano de gerenciamento de riscos efetivado, $27,3 \%$ assinalaram que às vezes há plano de gerenciamento de riscos efetivado ou em discussão e $10,1 \%$ assinalaram que não estabelecem plano de gerenciamento de riscos efetivamente.

O plano de gerenciamento dos riscos é o resultado desse primeiro processo e pode incluir diversos elementos como a estratégia dos riscos, a metodologia, os papéis e responsabilidades, o financiamento, os prazos, a categorias dos riscos, o apetite a riscos das partes interessadas, as definições de probabilidade e impacto dos riscos, a matriz de probabilidade e impacto, os formatos de relatórios e a forma de acompanhamento. (PMI, 2017)

dessa forma, nota-se que realizar essa etapa de forma efetiva e frequente é de extrema importância para criar e manter o gerenciamento dos riscos de forma organizada ao longo de qualquer projeto, sobretudo em projetos de implantação de uma rede elétrica inteligente (Silva, Oliveira e Silva, 2021).

Como foi apontado anteriormente, existem diversos parâmetros essenciais para implantação de SG e, quando comparada com a rede elétrica convencional, as redes elétricas inteligentes se destacam pela maior capacidade e complexidade, uma vez que existe uma maior quantidade de elementos envolvidos. Por isso, para gerir da forma mais eficiente e correta todos os diversos riscos que surgirão ao longo do projeto, é necessário realizar planejamento com frequência e de forma ativa.

\subsection{Identificação dos Riscos}

O segundo o processo Identificar dos Riscos é definido no Guia PMBOK (PMI,2017) como processo na qual é realizado a identificação dos riscos individuais do projeto, bem como fontes de risco geral do projeto, e de documentar suas características. Ainda no formulário, foi questionada a frequência e forma que é realizada a identificação dos riscos em cada organização, 63,6\% marcaram que sempre há identificação dos riscos de forma completa, 36,4\% disseram que há identificação dos riscos, mas de forma parcial e esporadicamente e, logo, nenhuma assinalou que não há efetivamente o processo de identificação dos riscos.

Utilizando então diversos elementos, entre eles o plano de gerenciamento de riscos criado no processo anterior, é realizada a identificação dos riscos gerando um registro e um relatório dos riscos com detalhes de cada risco identificado do projeto, apresentando inclusive informações sobre as fontes de risco geral e individual do projeto, além de atualizações de documentos do projeto como registro de premissas, registro das questões e o registro das lições aprendidas (PMI, 2017). Não há como, portanto, gerenciar os riscos sem ao menos identificá-los e, de acordo com as respostas obtidas, todos realizam essa identificação de alguma forma.

\subsection{Realizar a Análise Qualitativa dos Riscos}

O terceiro processo Realizar a Análise Qualitativa dos Riscos é descrito no Guia PMBOK como processo de 
priorização de riscos individuais do projeto para análise ou ação posterior, através da avaliação de sua probabilidade de ocorrência e impacto, assim como outras características. Dessa forma, foi feito questionamento sobre os tipos de técnicas utilizadas para realização da análise qualitativa dos riscos e como resposta obteve-se que 45,5\% realizam análise qualitativa dos riscos por técnicas estatísticas de ocorrências de impactos, 45,5\% fazem análise qualitativa dos riscos através da utilização de técnicas próprias e $9,1 \%$ não realizam análise qualitativa dos riscos.

Além do resultado principal, a concentração dos esforços nos riscos mais prováveis de ocorrerem, este processo estabelece as bases do processo Realizar a Análise Quantitativa dos Riscos e as prioridades relativas dos riscos individuais do projeto para o processo Planejar as Respostas aos Riscos. Logo, percebe-se sua imensa importância para as etapas seguintes do Gerenciamento dos Riscos do projeto. (PMI, 2017)

PMI (2017) destaca ainda que as avaliações realizadas nesse momento são subjetivas, pois se baseiam em percepções do risco pela equipe do projeto e outras partes interessadas, e para concluir uma avaliação eficaz é necessário uma identificação explícita e o gerenciamento das atitudes dos riscos dos participantes chaves durante esse processo. Dessa forma, mesmo existindo diversos motivos para escolher quais técnicas serão utilizadas para realização da análise qualitativa dos riscos, as técnicas estatísticas de ocorrências de impactos demonstram maior confiabilidade e clareza durante o processo ao invés das técnicas próprias desenvolvidas em algumas organizações.

\subsection{Realizar a Análise Quantitativa dos Riscos}

O quarto processo Realizar a Análise Quantitativa dos Riscos é definido pelo PMI (2017) como o processo de analisar numericamente o efeito combinado dos riscos individuais identificados no projeto e outras fontes de incerteza nos objetivos gerais do projeto. Foi realizado questionamento sobre os tipos de técnicas utilizadas para realização da análise quantitativa dos riscos e, 54,5\% afirmaram que há análise quantitativa dos riscos por técnicas que combinam riscos individuais, 27,3\% responderam que há análise quantitativa dos riscos por técnicas próprias e 18,2\% assinalaram que não há análise quantitativa dos riscos.

PMI (2017) afirma que nos projetos em que houver este processo, sua aplicação deve ocorrer ao longo de todo o projeto. Aponta ainda que a análise quantitativa de riscos é o único método confiável para avaliar o risco geral do projeto, utilizando a avaliação do efeito agregado de todos os riscos individuais e outras fontes de incerteza sobre os resultados do projeto. Sendo assim, realizar essa análise utilizando técnicas próprias não é

um método confiável por não relacionar todos os riscos individuais e outras fontes de incerteza com os resultados esperados nos projetos de implantação de redes elétricas inteligentes.

\subsection{Planejar das Respostas aos Riscos}

O quinto processo Planejar das Respostas aos Riscos é descrito no Guia PMBOK como o processo de desenvolver alternativas, selecionar estratégias e acordar ações para lidar com a exposição geral de riscos e tratar os riscos individuais do projeto. Como resultado, 64,5\% assinalaram que sempre há planos de respostas a riscos efetivados, 27,3\% que há planos de respostas a riscos feitos de forma parcial e em alguns projetos e 18,2\% marcaram que nunca há plano de respostas a riscos efetivado.

Ao longo do gerenciamento de riscos, os riscos são identificados, analisados, priorizados e então planos são criados pelos responsáveis para resolver cada risco de forma adequada de acordo com a relevância do risco, custos, contexto do projeto e aprovado por todas as partes envolvidas. Este processo deve ser realizado também ao longo de todo o projeto e como principal benefício obtém-se a identificação de formas apropriadas de abordar o risco geral e os riscos individuais do projeto.

(PMI, 2017) 
Não realizar nenhum plano de respostas aos riscos, ou realizar apenas de forma parcial ou em alguns projetos indica uma deficiência no gerenciamento dos riscos das organizações analisadas neste presente trabalho. Acarretando, consequentemente, em maiores dificuldades para implementação das respostas.

\subsection{Implementar das Respostas aos Riscos}

O sexto processo Implementar das Respostas aos Riscos é definido no Guia PMBOK como o processo de implementação de planos acordados de resposta aos riscos. Após questionamento sobre a frequência e forma que é realizada a implementação das respostas aos riscos, 45,5\% assinalaram que sempre há a implementação de respostas aos riscos efetivamente, 45,5\% afirmaram que há implementação de respostas aos riscos de forma parcial ou alguns projetos e 9,1\% marcaram que não há a implementação de respostas aos riscos.

Ao final deste processo podem ser solicitadas mudanças nas linhas de base de custos e do cronograma ou de outros componentes do plano de gerenciamento do projeto, bem como a atualização em alguns documentos como o registro das questões, o registro das lições aprendidas, as atribuições da equipe do projeto, o registro dos riscos e o relatório de riscos. (PMI, 2017) A não implementação das respostas aos riscos resultaria por tanto na falta de controle dos riscos.

Nota-se que dentre as seis que sempre realizam planejamento de respostas aos riscos, cinco sempre implementam as respostas e um implementa de forma parcial ou em alguns projetos. Além disso, em uma organização não é realizado o planejamento das respostas, mas elas são mesmo assim implementadas de forma parcial ou em alguns projetos. Apenas um respondente sinalizou que em seus projetos envolvendo redes elétricas não são planejadas e nem implementadas respostas aos riscos de nenhuma forma.

\subsection{Monitorar os Riscos}

O sétimo e último processo Monitorar os Riscos é descrito no Guia PMBOK como o processo de monitorar a implementação de planos acordados de resposta aos riscos, acompanhar riscos identificados, identificar e analisar novos riscos, e avaliar a eficácia do processo de risco ao longo do projeto. Como resultado, 63,6\% disseram que sempre há o monitoramento dos riscos efetivamente e 36,4\% afirmaram que há o monitoramento dos riscos de forma parcial ou nunca é uma prioridade na execução, ou seja, nenhum assinalou que não há o monitoramento dos riscos.

Ao longo deste processo são geradas informações sobre o desempenho do trabalho, solicitadas mudanças nas linhas de base dos custos e cronograma ou outros componentes do plano de gerenciamento do projeto, bem como atualização dos documentos (registro de premissas, registro das questões, registro das lições aprendidas, registro dos riscos e o relatório de risco) e atualização nos ativos de processos organizacionais (modelos do plano de gerenciamento dos riscos, estrutura analítica dos riscos, registro e relatório de riscos). (PMI, 2017)

Realizar todos os outros sete processos referentes ao Gerenciamento de Riscos e não realizar o monitoramento dos riscos torna praticamente inviável a continuidade do projeto de forma satisfatória, visto que não seriam geradas as informações sobre o desempenho do trabalho e nem poderiam ser solicitadas as mudanças e atualizações necessárias das linhas de bases, do plano de gerenciamento do projeto, documentos e ativos de processos organizacionais. Destaca-se, portanto, a imensa importância da efetividade da monitoração dos riscos.

\section{Discussão}

Reunindo todos os grupos de processos em um mesmo gráfico de colunas, obteve-se a Figura 1 a seguir que demonstra a classificação da intensidade dos riscos em cada grupo de processos ao longo dos projetos que envolvem energia elétrica do ponto de vista das organizações do setor privado. Ainda, para melhor visualização, o gráfico de colunas foi 
reconstruído em um gráfico de linhas (senoidal) que apresenta de forma contínua a implementação e preocupação com gestão de risco durante o ciclo de vida do projeto, disposto na Figura 2 em sequência.

Figura 1 - Classificação da intensidade dos riscos em cada grupo de processos (gráfico de colunas).

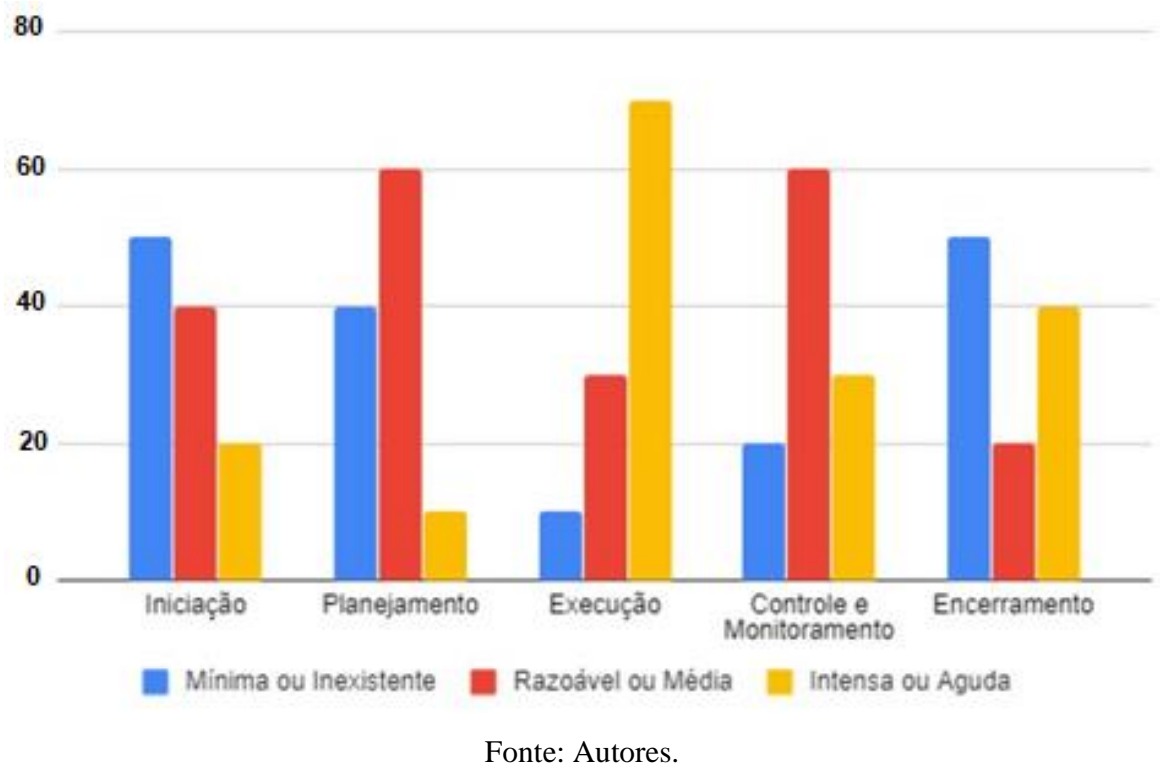

Como é possível observar a intensidade da gestão de riscos em cada fase sofre variações conforme a percepção ilusória de que apenas na execução o risco é mais eminente.

Figura 2 - Variação da intensidade dos riscos em cada grupo de processos constante (gráfico senoidal).

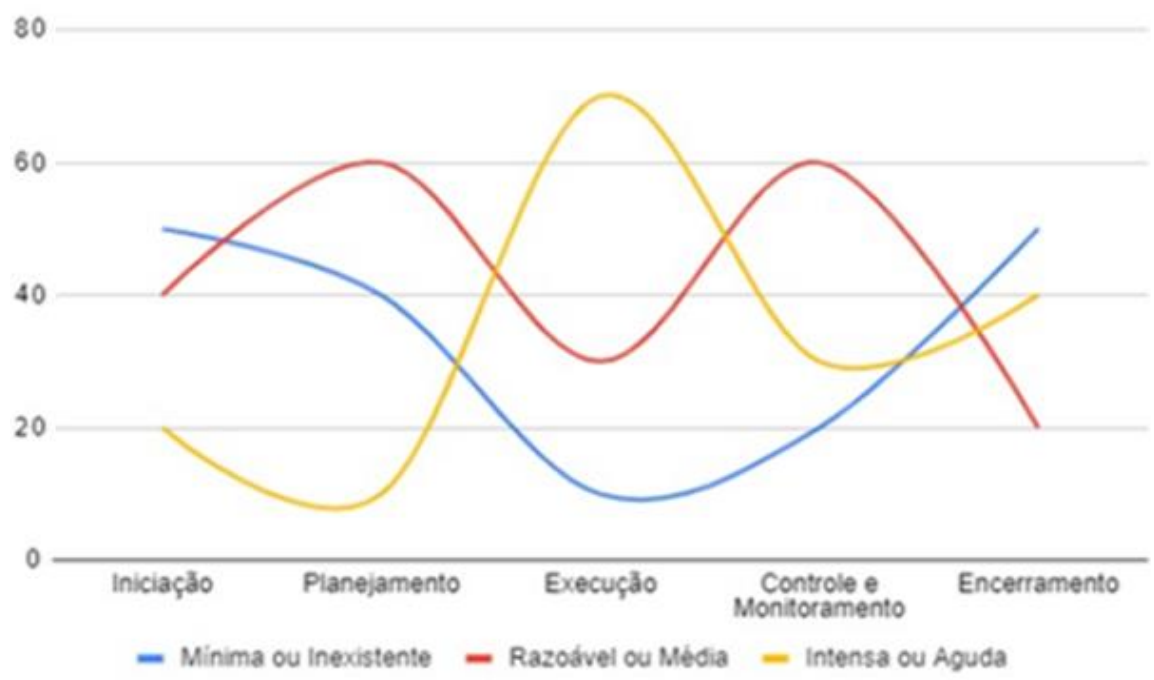

Fonte: Autores.

A intensidade de riscos, nomeada como Mínima ou Inexistente, é identificada por uma forma geométrica muito semelhante a uma parábola com concavidade para cima. Segundo os resultados obtidos, o grupo da Iniciação e Encerramento apresentaram maior preocupação por parte dos participantes, o Planejamento vem em seguida e o Controle e Monitoramento em posteriormente. A Execução se enquadra como o vértice da parábola, é tida como de intensidade Mínima ou Inexistente pela minoria, o que sugere uma aplicação de recursos maior nesse grupo, pois como se poderá observar adiante, a Execução é o grupo com maior intensidade. 
A classificação Razoável ou Média para a intensidade de riscos foi a mais apontada, com destaque para os grupos de Planejamento e de Controle e Monitoramento que foram os que obtiveram maior destaque para essa categoria. Em seguida, em ordem decrescente se têm a Iniciação, a Execução e, por último, o Encerramento. O que remete a uma compreensão sobre um equilíbrio na intensidade das preocupações com os riscos nesses grupos.

O último cenário de aplicação Intensa ou Aguda de intensidade de riscos tem forma geométrica semelhante a uma parábola com concavidade para baixo. O grupo Execução foi o que teve maior destaque, seguido dos demais grupos analisados. Refletindo a preocupação dos profissionais que participaram em investir mais recursos na gestão de risco desse grupo Execução com objetivo de minimizar os efeitos de uma eventual falha no projeto.

\section{Conclusão}

Todos os processos de gerenciamento dos riscos do projeto são apresentados como processos discretos com interfaces definidas no Guia PMBOK (PMI,2017), mas na prática há sobreposição, tornando assim impossível detalhá-los completamente.

Foi observado que embora que os profissionais tenham vasta experiência no setor elétrico, ainda possuem um conhecimento superficial sobre os reais riscos que uma arquitetura de uma Redes Elétricas Inteligentes está submetida, por isso, boa parte das respostas obtidas foram baseadas nas experiências destes profissionais em redes elétricas convencionais.

Além de bem instruídos, os profissionais participantes da pesquisa possuem altos cargos nas organizações em que atuam e, a maior parte possui como local da lotação o estado de São Paulo (SP). Dentre as áreas de atuação das organizações, a comercialização de energia elétrica e consultoria e a assessoria no setor elétrico foram as mais apontadas, atuando principalmente no domínio nacional.

Também foi observado que apenas dois processos, identificação e monitoramento dos riscos, são amplamente realizados pelas organizações. Acredita-se que isso ocorra uma vez que não há como gerenciar os riscos sem ao menos identificá-los e monitorá-los de alguma forma. Em contrapartida, a análise quantitativa dos riscos e o planejamento das respostas aos riscos são os processos menos realizados por parte das organizações privadas, indicando assim uma possível dificuldade de avaliação do risco geral do projeto e de implantação das respostas aos riscos.

O planejamento do gerenciamento dos riscos, processo inicial do Gerenciamento de Riscos, quase sempre ocorre de forma efetiva, embora algumas organizações não realizem esse processo ou apenas realizem às vezes de forma efetivada. Devido a maior capacidade e complexidade das Redes Elétricas Inteligentes, recomenda-se que, para gerir da forma mais eficiente e correta todos os diversos riscos que surgirão ao longo do projeto, seja efetuado o planejamento frequentemente e efetivamente.

Já a análise qualitativa dos riscos quase sempre ocorre, mas, quando é posta em prática, apenas metade das organizações utiliza técnicas que demonstram maior confiabilidade e clareza. É importante ressaltar que mesmo existindo diversos motivos para escolher quais técnicas serão utilizadas para realização da análise qualitativa dos riscos, as técnicas estatísticas de ocorrências de impactos demonstram maior confiabilidade e clareza durante o processo ao invés das técnicas próprias desenvolvidas em algumas organizações.

A implantação das respostas aos riscos apresenta comportamento semelhante à análise qualitativa dos riscos. Quase sempre são implementadas as respostas, mas, quando ocorre, apenas metade das organizações realiza de forma frequente e efetiva, ocasionando na falta de controle eficaz dos riscos nas organizações que não implantam as respostas ou implantam de forma parcial ou em alguns projetos.

Já que no Planejamento do Projeto são realizados cinco dos sete processos do Gerenciamento de Riscos (Planejamento do Gerenciamento dos Riscos, Identificação os Riscos, Realizar a Análise Qualitativa dos Riscos, Realizar a 
Análise Quantitativa dos Riscos e Planejar as Respostas), nota-se, portanto, que se trata da fase que pode apresentar maiores complicações caso não seja desenvolvida de forma satisfatória e prejudicando ainda o restante dos grupos de processos.

de acordo os participantes, durante a Execução do Projeto a intensidade dos riscos se dá de forma intensa ou aguda, sendo que é nessa fase que ocorre o processo de Implementação das Respostas aos Riscos. Porém, a Execução do Projeto só demonstrará maior intensidade de riscos se o Planejamento do Projeto não for cumprido de forma efetiva, completa e utilizando técnicas e ferramentas confiáveis e bem difundidas. Portanto, os riscos podem vir a se manifestar de forma mais intensa durante a Execução do Projeto, de forma razoável no Planejamento e no Controle e Monitoramento do Projeto e de forma mínima ou inexiste na Iniciação e no Encerramento do Projeto.

Enfim, a partir deste estudo realizado sobre o gerenciamento de riscos em projetos de redes elétricas inteligentes, pode-se concluir que as organizações do setor privado analisadas através do conhecimento e experiências de seus profissionais, em sua maioria, realizam mais intensidade o gerenciamento de riscos, embora nem sempre de forma frequente, completa e efetiva ou ainda utilizando técnicas e ferramentas altamente confiáveis e difundidas.

Como trabalho futuro, se deseja analisar os impactos financeiros e econômicos nos projetos de Redes Elétricas Convencionais, que não planejaram e executaram adequadamente uma gestão de riscos em seus projetos.

\section{Referências}

Alves, L. M., Souza, G., Ribeiro, P., \& Machado, R. J. (2021). Longevity of Risks in Software Development Projects: a Comparative Analysis With an Academic Environment. Procedia Computer Science, 10.1016/j.procs.2021.01.236, 181, (827-834).

Arruda, M. V. N. \& Oliveira, R. (2013). Implementação de Projetos Smart Grid No Brasil. In: Anais Jornada de Pesquisa e Extensão. Cuiabá: Instituto Federal de Educação, Ciência E Tecnologia de Cuiabá. http://Jornada.Cba.Ifmt.Edu.Br/Jornada/Index.Php/Jornada2013/Jornada2013/Paper/View/78>.

Bandeira, F. P. M. (2012). Redes de Energia Elétrica Inteligentes. Nota Técnica. Consultoria Legislativa. Câmara dos Deputados.

Babbie, E. (2005). Métodos de Pesquisa de Survey. UFMG. 519p.

Boccuzzi, C. V. (2010). Smart Grid e o Big Brother Energético. Metering International América Latina, 3:82-83.

Carnevalli, J.A. \& Miguel, P.A.C (2001). Desenvolvimento Da Pesquisa de Campo, Amostra e Questionário Para Realização de Um Estudo Tipo Survey Sobre A Aplicação do OFD No Brasil. Anais. ENEGEP - XXI Encontro Nacional de Engenharia de Produção, Salvador.

Cavalcante, N. W. F. et al (2019). Casos Para Ensino: Smart Grid na América Latina: Caso Ampla de Inovação no Setor Elétrico. Revista de Administração Contemporânea, Maringá, V. 23, N. 3, P.416-435.

Costa, H. A. de O., Gomes, L. L., Costa, D. C. L., Rocha, E. M., Francês, C. R., \& Andrade, S. H. (2021). Fractional order differential calculus applied on decision making system to smart grid management via decision trees. Research, Society and Development, $10(16)$, e38101623387. https://doi.org/10.33448/rsd-v10i16.23387

Fernandes, N. C. et al (2013). Smart Grid Para o Sistema Elétrico: IEEE. 134 Slides.

Filippetto, A. S. et al (2021). A Risk Prediction Model For Software Project Management Based On Similarity Analysis Of Context Histories. Information And Software Technology Journal, 131.

Firmes, V. P., \& Celeste, W. C. (2018). A bibliometric review on identifying similar loads in Smart Grid. Research, Society and Development, 7(12), e11712497. https://doi.org/10.33448/rsd-v7i12.497

Gallotti, V. D. M. (2021). Intelligent electric power networks (Smart Grids). Research, Society and Development, 10(9), e30010918322. https://doi.org/10.33448/rsd-v10i9.18322

Gil, A. C. (2007). Como Elaborar Projetos de Pesquisa. (4a ed.). Editora Atlas.

Guerra, J. et al (2010). Smart Grid é o Futuro Certo da Distribuição de Energia Elétrica? (5a ed.). Portal O Setor Elétrico. http://www.instalacoeseletricas.com/download/smart_grid.pdf.

Kerzner, H. (2001). Project Management: A System Approach to Planning Scheduling and Controlling. John Wiley \& Sons, (7a ed.).

Marconi, M. D. A. \& Lakatos, E. M. (1996). Técnicas de Pesquisa: Planejamento e Execução de Pesquisas, Amostragens e Técnicas de Pesquisas, Elaboração, Análise e Interpretação de Dados. Editora Atlas. (3a ed.).

Mattar, F. N (1996). Pesquisa de Marketing: Edição Compacta. Editora Atlas. 
Research, Society and Development, v. 10, n. 17, e243101724947, 2021

(CC BY 4.0) | ISSN 2525-3409 | DOI: http://dx.doi.org/10.33448/rsd-v10i17.24947

MME - Ministério de Minas e Energia. (2010). Relatório Smart Grid - Grupo de Trabalho de Redes Elétricas Inteligentes. http://antigo.mme.gov.br/documents/36148/342584/relat\%c3\%93rio+smart+grid/cf509d1b-b503-5eda-5392-97738fe6f45a?version=1.0

Oliveira, G. A. Q. S., Cleto, M. G., \& Seleme, R. (2015). Como Avaliar Projetos de Implementação de Smart Grids: Uma Revisão Sistemática. Iberoamerican Journal of Project Management (IJOPM), 6(1), 47-78.

Pereira, Guilherme, Weiss, Mariana (2017). Redes Inteligentes: Principais Desafios Para o Caso Brasileiro. FGV Energia, 4-6.

PMI - Project Management Institute (2017). Um Guia Do Conhecimento Em Gerenciamento de Projetos. Guia PMBOK®. (6a ed.).

SIMA - Secretaria de Infraestrutura e Meio Ambiente (2015). Perdas Do Furto de Energia. http://www.energia.sp.gov.br/2017/03/furto-de-energia-causaperda-de-r-8bilhoes-em-2015-estima-acende-brasil.

Shabanzadeh, M. \& Moghaddam, M. P. (2013). What is The Smart Grid? Definitions, Perspectives, And Ultimate Goals. 28th Power System Conference Tehran, Iran.

Silva, G. G., Oliveira, K. R. \& Silva, J. F. R. (2021). Maturidade no Gerenciamento de Riscos em Projetos de Smart Grid no Setor Privado Brasileiro. Anais do XXVIII Simpósio de Engenharia de Produção da Unesp. https://www.simpep.feb.unesp.br/anais.php.

Silva, S. M., Santos, C. C. M. \& Siqueira, J. O. (1998). O Uso Do Questionário Eletrônico Na Pesquisa Acadêmica: Um Caso de Uso Na Escola Politécnica Da Universidade de São Paulo. Anais. South Padre Island: Balas/University of Texas. 